\title{
Research and Analysis of Electromagnetic Noise of Outdoor SVG Power Unit
}

\author{
Xiaobo Jiang1, Fengli Han2, Tao Liu3 \\ 1China North Engine Research Institute(Tianjin),Tianjin,300400,China \\ 2Shenyang Power Supply Company, Liaoning Electric Power Company \\ Limited, State Grid, Shenyang, Liaoning 110003 \\ 3State Grid Liaoning Electric Power Supply Co., Ltd. Fuxin Power \\ Supply Branch, Fuxin, Liaoning 123000, China
}

\begin{abstract}
Outdoor SVG (container-type) is especially suitable for limited space, such as old substations. The limited space of the entire device, and electromagnetic noise inside, especially that of the power chamber which is far higher than ordinary SVG. In the final analysis, the study on the law of change of SVG power chamber electromagnetic noise is the study on the law of change of IGBT current and voltage inside the power unit of the power chamber. Due to the complex structure of IGBT, the change of variants is usually nonlinear. Therefore, electromagnetic noise cannot be analyzed with common algorithms. In this paper, a method of differential mode and common mode electromagnetic noise spectrum calculation through analysis of dynamics of IGBT inside the power unit at different stages and derivation of law of change of current and voltage at different stages is introduced.
\end{abstract}

Keywords: outdoor SVG, differential mode noise, common mode noise Matlab

\section{Introduction}

Now $10 \sim 35 \mathrm{kV}$ SVG is generally of chain structure in China, which means commutation chain of each phase is formed by connecting power units. The end voltage of each power unit is up to $680 \mathrm{~V}$, and current up to $279 \mathrm{~A}$. Due to frequent switching under high current and voltage, the variation ratios of current and voltage of these devices are high. Noise signals can be conducted or radiated via the parasitic inductor and parasitic capacitor of the line or the element, affecting the normal functioning of other precise electronic devices in surrounding area and even the neighbor power units. Taking SVG $(10 \mathrm{kV} \pm 5 \mathrm{M})$ of a model for example, each phase of commutation chain consists of 12 power units in serial connection. The entire power chamber has 36 power units which are compactly arranged, with space of $222 \mathrm{~mm}$. 


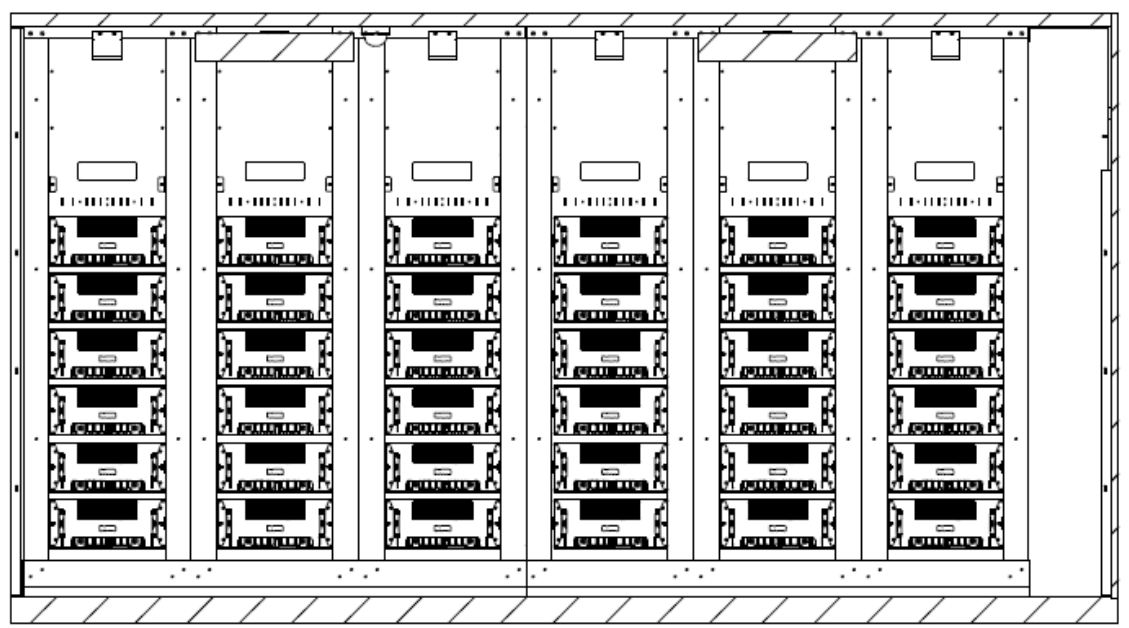

Fig.1. Spatial layout of outdoor SVG (container-type) power chamber

In this layout, the electromagnetic noise of outdoor SVG (container-type) must be focused on, while that generated by the power module is even more crucial.

\section{Method for analysis of unit electromagnetic noise of SVG power}

Generally speaking, the intense change in current and voltage in power module during IGBT switching is the most important reason for electromagnetic noise generation. Therefore, the law of change of the current and voltage during IGBT switching should be the focus. Given the complex structure of IGBT, a controlled current source based IGBT dynamic analyzing equivalent circuit with parasitic capacitor at the port is built to analyze IGBT dynamics. 


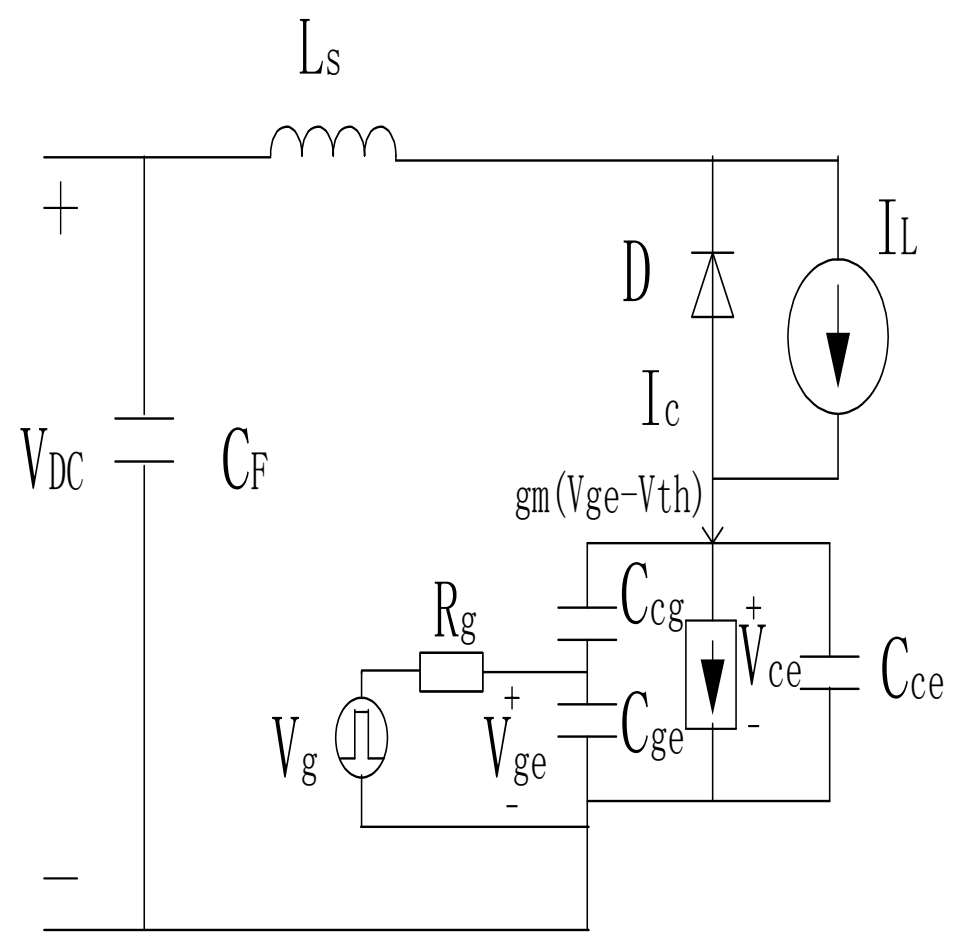

Fig. 2. IGBT dynamic analyzing equivalent circuit

\section{Analysis of IGBT switch-on in power module}

IGBT switch-on is divided into four stages: Stage I ( ${ }^{21}$ ), grid driving voltage turns positive from negative, and grid driving voltage rises to grid threshold. Stage II ( $\tau 2$ ), collector current starts to rise and reaches the maximum. At this stage, the collector current starts to change at the rate of

$$
\frac{d i_{c}}{d t}(\tau 2)=g m * \frac{\left(V_{g+}-V_{t h}\right)}{R_{g} C_{i e s}+L_{s} g m}
$$

(1)

At this time, the collector voltage drops at the rate of: $\frac{d V_{c e}}{d t}(\tau 2)=-\frac{L s}{\tau 3} * \frac{d i c}{d t}(\tau 2)$

Stage III ( $\tau 3$ ), collector current ic drops from the maximum to the a stable level at the rate of:

$$
\frac{d i_{c}}{d t}(\tau 3)=-\frac{1}{\tau 3} * \sqrt{2 \tau_{L T} I_{L} \frac{d i_{c}}{d t}(\tau 2)}
$$

(3) 
Meanwhile, collector voltage drops to on-state voltage at a rate of:

$$
\frac{d V_{c e}}{d t}(\tau 3)=-\left(\frac{V_{g+} g m-V_{t h} g m-I_{L}}{R_{g} C_{c g} g m}+\frac{C_{g e}}{C_{c g} g m} \frac{d i_{c}}{d t}(\tau 2)\right)
$$

(4)

It can be seen that Formula (4) mainly consists of the variation rates of the gate pole current and the collector current at Stage II. Besides, Miller effect causes huge equivalent input capacitance, leading to appearance of voltage platform at grid emitting pole. The current of the grid emitting pole is almost completely injected into the gate collecting pole capacitor,

i.e. $\quad i_{g} \approx C_{c g} \frac{d V c e}{d t}$, leading to $\frac{d V_{c e}}{d t}(\tau 3) \approx \frac{V_{D D}-V_{g e(M)}}{C_{c g} R_{g}}$

Stage IV ( ${ }^{\tau 4}$ ), Miller effect disappears, the gate collecting pole capacitor voltage starts to grow, Ciss starts charging, and collector voltage and current basically remain the same.

\section{Analysis of IGBT switch-off in power module}

IGBT switch-off is also divided into four stages. Stage I ( $\tau 1^{\prime}$ ), at the beginning, collector voltage grows slowly, and so does Ccg. Stage II ( $\left.\tau 2^{\prime}\right), C_{c g}$ reaches a stable level and the collector voltage rapidly grows at the rate of:

$$
\frac{d V_{c e}}{d t}\left(\tau 1^{\prime}\right) \approx \frac{V_{t h}-V_{g-}+I_{L} / g m}{C_{c g} R_{g}}
$$

$$
\begin{aligned}
& \text { The corresponding current ic changes at a rate of: } \\
& \frac{d i c}{d t}\left(\tau 1^{\prime}\right)=-\frac{L s}{\tau 2} * \frac{d i c}{d t}\left(\tau_{2}\right) \text { (7) }
\end{aligned}
$$

Stage III ( $\tau 3^{\prime}$ ) , diode is quickly switched on, collector current drops rapidly at a

rate

of

$$
\frac{d i_{c}}{d t}\left(\tau_{2}^{\prime}\right)=\frac{\left(g m V_{g-}-V_{t h}-I_{L} / g m\right)}{R_{g} C_{i e s}+g m L_{s}}
$$

$$
\text { Voltage changes at a rate of } \frac{d V_{c e}}{d t}\left(\tau_{3}{ }^{\prime}\right)=-\frac{L s}{\tau_{3}{ }^{\prime}} * \frac{d i c}{d t}\left(\tau_{2}{ }^{\prime}\right)
$$

(9)

Stage IV ( $\left.\tau 4^{\prime}\right)$, IGBT enters the trailing period. Voltage Vge drops to the switch-off level. IGBT enters a switch-off status. The collector voltage and current remain unchanged. 


\section{Differential mode noise and common mode noise in power module}

In electrical and electronic field, people generally divide electromagnetic noise into differential mode noise and common mode noise, wherein the differential mode noise is caused by the pulsating current of the switch converter while the common mode noise is caused by the interaction between the voltage change of the switch converter and the stray parameters of the device. For SVG, differential mode noise in power unit is generated during IGBT switching when the current variation $\mathrm{di} / \mathrm{dt}$ acts on the parasitic inductor inside; common mode noise is generated during IGBT switching when the voltage variation $\mathrm{dv} / \mathrm{dt}$ acts on the parasitic capacitor between the circuit and the ground. Based in formulas (1) (9) got through analyzing the law of change of current and voltage during IGBT switching, we can estimate the intensity and spectrum of differential mode noise and common mode noise in the power unit as follows:

1. Look up the user manual to get the basic coefficients needed for calculation based on formulas (1) (9) according to the IGBT model in the power unit, such as Ccg,Cies.

2. Perform Fourier transform for formulas $(1) \sim(9)$ to get their frequency domain formulas $(1) \sim(9)$.

3. Use Matlab to build differential mode and common mode noise circuit model according to the electrical parameters and actual structure of the power unit.

4. Perform frequency domain analysis for differential mode and common mode noise circuit to get the spectrums of the differential mode and the common mode noise of the power unit.

\section{Conclusion}

Due to the complex structure of IGBT, differential mode and common mode electromagnetic noise in power unit cannot be analyzed correctly with conventional electromagnetic noise analysis methods, so that the designer cannot evaluate the electromagnetic compatibility of the entire outdoor SVG, thus affecting the reliability of the equipment.

Now the mathematical formulas of the current voltage variation (noise source) are deducted based on the main stages during switching of IGBT inside the power unit. Matlab is used to get electromagnetic noise spectrum and modify the electrical structure of the electrical structure of the outdoor SVG power chamber, thus reducing the impact of electromagnetic noise on the power chamber and even the entire device. 


\section{References}

[1] Larsen E V, Miller N W ,Nilsson S L etal. Benefits of GTO-based compensation systems for electric utility applications[J].IEEE Trans on Power Delivery,1992,7(4):2056-2064

[2] K.Frank, J.Petzodlt, H.Volker. Experiment and simulative investigations of conducted EMI Performance of IGBTs for 5-10KVA onverters[C].IEEE PESC 1996,pp.1986-1991.

[3] I.Zverev,S.Konrad,H.Voelker,J.Petzoldt,F.Kltz.Influence of the gate drive techniques on the conducted EMI behavior of a power cinverter[C].IEEE PESC 1997,pp.1522-1528.

[4] Xuejun Pei, Kai Zhang,Yong Kang,et al.Prediction of common mode conducted EMI in single phase PWM inverter[J].In: 35th Annual Power Electronics Specialists Conference, PESC 04.USA:IEEE,20-25 June,2004.3060-3065. 